Pesq. Vet. Bras. 37(3):205-209, março 2017 DOI: $10.1590 / \mathrm{S} 0100-736 \mathrm{X} 2017000300001$

\title{
Diagnóstico e fatores de risco do complexo teníase-cisticercose bovina no município de Salinas, Minas Gerais ${ }^{1}$
}

\author{
Fernanda C. Magalhães², Thiago M. Santos ${ }^{3}$, Débora C. Assis², Cleia D. Ornellas², \\ Paulo A. Pinto ${ }^{4}$ e Wagner M. Santos ${ }^{2 *}$
}

\begin{abstract}
Magalhães F.C., Santos T.M., Assis D.C., Ornellas C.D., Pinto P.A. \& Santos W.M. 2017. [Diagnosis and risk factors of bovine taeniasis-cysticercosis complex in Salinas, Minas Gerais, Brazil.] Diagnóstico e fatores de risco do complexo teníase-cisticercose bovina no município de Salinas, Minas Gerais. Pesquisa Veterinária Brasileira 37(3):205209. Departamento de Tecnologia e Inspeção de Produtos de Origem Animal, Escola de Veterinária, Universidade Federal de Minas Gerais, Av. Antônio Carlos 6627, Belo Horizonte, MG 30123-970, Brazil. E-mail: wagnerlmds@ufmg.br

In order to diagnose the situation of bovine taeniasis-cysticercosis complex in the municipality of Salinas, Minas Gerais, Brazil, blood samples were collected from 355 cattle in 18 randomly selected farms. A socioeconomic questionnaire was filled in each farm for the analysis of factors which favor the maintenance of the taeniasis-cysticercosis complex. An epidemiological survey of human taeniasis was performed through analyses of the Municipal Health Department in the 2007-2010 period. A prevalence of $4.7 \%$ for bovine cysticercosis and the frequency of $0.29,0.36,0.24$ and $0.24 \%$ for human taeniasis, during the evaluated period, was found. Among the risk factors, a statistically significant correlation was found between the occurrence of bovine cysticercosis and the ingestion of undercooked meat. It was concluded that bovine cysticercosis is present in the municipality of Salinas, due to inefficient heat treatment of the meat as the main risk factor for maintenance of the taeniasis-cysticercosis complex, reinforcing the need to adopt control measures with continuous epidemiological and health surveillance.
\end{abstract}

INDEX TERMS: Bovine, cysticercosis, taeniasis, ELISA, Immunoblot, Salinas.

RESUMO.- Com o objetivo de diagnosticar a situação do complexo teníase-cisticercose bovina no município de Salinas, Minas Gerais, foram coletadas amostras de sangue de 355 bovinos distribuídos em 18 propriedades rurais, sorteadas aleatoriamente. Em cada propriedade, foi aplicado um questionário socioeconômico para a análise de fatores que favorecem a manutenção do complexo teníase-cisticercose bovina. Foi realizado também um levantamento epidemiológico dos casos de teníase diagnosticados nos laboratórios credenciados pela Secretaria Municipal de Saúde de Sa-

\footnotetext{
${ }^{1}$ Recebido em 23 de setembro de 2015.

Aceito para publicação em 1 de agosto de 2016.

${ }^{2}$ Departamento de Tecnologia e Inspeção de Produtos de Origem Animal, Escola de Veterinária, Universidade Federal de Minas Gerais (UFMG), Av. Antônio Carlos 6627, Belo Horizonte, MG 30123-970, Brasil. *Autor para correspondência: wagnerlmds@ufmg.br

${ }^{3}$ Instituto Federal do Norte de Minas Gerais (IFNMG), Campus Salinas, Fazenda Varginha Rodovia MG-404 Km 2 s/n, Zona Rural, Salinas, MG 39560-000, Brasil.

${ }^{4}$ Departamento de Medicina Veterinária, Universidade Federal de Viçosa (UFV), Av. P.H. Rolfs s/n, Viçosa, MG 36571-000, Brasil.
}

linas, no período de 2007 a 2010. A prevalência de cisticercose bovina foi de $4,70 \%$ enquanto as prevalências de teníase, encontradas durante os quatro períodos avaliados, foram de $0,29 \%, 0,36 \%, 0,24 \%$ e $0,24 \%$. Entre os fatores de risco para a manutenção do complexo teníase-cisticercose analisados, foi observada uma relação estatisticamente significativa entre a ocorrência de cisticercose bovina e a ingestão de carne malpassada pelos entrevistados. Foi concluído que a cisticercose bovina está presente no município de Salinas, Minas Gerais, sendo o tratamento térmico ineficiente da carne bovina o principal fator de risco para a manutenção do complexo teníase-cisticercose, o que reforça a necessidade da adoção de medidas de controle com contínua vigilância epidemiológica e sanitária.

TERMOS DE INDEXAÇÃO: Bovino, cisticercose, teníase, ELISA, Immunoblot, Salinas.

\section{INTRODUÇÃO}

O complexo teníase-cisticercose pode ser definido como um conjunto de alterações patológicas provocadas pelas 
formas adultas e larvares de Taenia saginata e Taenia solium. Estas zoonoses podem se expressar sob duas formas distintas, sendo caracterizada por uma parasitose intestinal, a teníase, que acomete o homem, ou por uma parasitose extra-intestinal, a cisticercose, provocada pela presença da forma larvar do parasita nos tecidos de bovinos, suínos ou do próprio homem.

O complexo teníase-cisticercose é de distribuição mundial, ocorrendo principalmente em países em desenvolvimento, onde existem grupos ou populações humanas convivendo com animais sob condições que favorecem a transmissão e manutenção do ciclo zoonótico. De acordo com a Organização Mundial da Saúde (OMS), este complexo faz parte do grupo das "doenças da pobreza" e continua sendo negligenciada principalmente devido à ausência de informação sobre a sua distribuição geográfica e falta de recursos para o seu controle. A ocorrência dessas doenças em áreas com menor desenvolvimento econômico é favorecida por apresentarem condições precárias de saneamento básico e baixo nível de desenvolvimento socioeconômico, podendo estar associada também à ausência de sistemas de criação de gado adequados, condições inadequadas de abate, bem como à falta de inspeção sanitária dos animais abatidos (Almeida et al. 2002, Flisser et al. 2003, Silva \& Silva 2007).

Segundo dados da OMS, já foram estimados, aproximadamente, 2,5 milhões de pessoas acometidas por teníase, distribuídas principalmente na América Latina, União Soviética, África, Índia e Extremo-Oriente (OPAS 1994). Outros dados apontam a neurocisticercose como responsável por 50.000 óbitos por ano em países subdesenvolvidos. No Brasil, os estados de São Paulo, Minas Gerais, Paraná e Goiás apresentam a doença em forma endêmica (Mendes et al. 2005). A Organização Pan-Americana de Saúde (OPAS 1994) estabeleceu como níveis que caracterizam endemicidade, a prevalência de $1 \%$ para teníase humana, $0,1 \%$ para cisticercose humana e 5\% para cisticercose animal. A teníase foi registrada em Minas Gerais, Brasil, com prevalência de $0,2 \%(185 / 100.144)$ obtida por meio de inquérito epidemiológico em população humana do Triângulo Mineiro (Esteves et al. 2005).

Taenia saginata, apesar de não causar a cisticercose em humanos, possui grande importância em saúde pública devido à morbidade provocada pela teníase. Além dos riscos à saúde pública, esta zoonose também acarreta prejuízos econômicos, sendo a principal causa de condenação de bovinos abatidos em frigoríficos sob Inspeção Veterinária Oficial (Marques et al. 2008, Khaniki et al. 2010, Vieira et al. 2011). Os dados estatísticos do abate desses bovinos demonstraram, em diversos levantamentos, uma prevalência de cisticercose variando de 0,32\% a 6,86\% entre anos de 1990 a 1997 (Reis et al. 1996, Moreira et al. 2002). Entretanto, a metodologia utilizada em tais levantamentos apresenta certas limitações, pois os dados obtidos são provenientes dos registros de lesões observadas durante a inspeção post-mortem dos animais abatidos, e, devido à baixa sensibilidade do exame, muitos casos positivos não são diagnosticados, especialmente aqueles em que a infecção é baixa e moderada, situação frequente no caso da cisticercose bovina. Além disso, nesses levantamentos, não se considera o grande número de animais destinados a abates não inspecionados (Almeida et al. 2002, Silva \& Silva 2007). Dessa maneira, métodos diagnósticos mais precisos para a detecção dos animais infectados, independentemente do grau de infecção e da localização do cisto no organismo do animal devem ser desenvolvidos, visando o aumento da eficiência do diagnóstico de cisticercose.

As metodologias Enzyme-Linked Immunosorbent Assay (ELISA) e Immunoblot têm sido utilizadas experimentalmente para o diagnóstico de cisticercose suína e humana, empregando extratos antigênicos de larvas de Taenia saginata, T. solium e T. crassiceps. Os testes ELISA e Immunoblot se complementam no diagnóstico da cisticercose, devido à alta sensibilidade e aplicabilidade do ELISA, que pode ser utilizado como um método de triagem, e à alta especificidade do Immunoblot, que pode ser utilizado para a confirmação dos resultados positivos no ensaio de ELISA (Pinto et al. 2000, Pinto et al. 2001, Sato et al. 2003, Dorny et al. 2003, Monteiro et al. 2007). 0 emprego destas metodologias no diagnóstico da cisticercose bovina pode contribuir para aumentar a precisão dos dados oficiais em estabelecimentos inspecionados de abate, além de ser uma ferramenta importante para a coleta de dados em estudos epidemiológicos e monitoramento de parasitoses em animais de abate (Girotto et al. 2009, Santos et al. 2013).

Com base nestes aspectos, os objetivos deste trabalho foram determinar a prevalência real de propriedades e animais positivos para a cisticercose bovina, avaliar a prevalência de teníase humana e avaliar as condições socioeconômicas e sanitárias que poderiam influenciar na transmissão do complexo teníase-cisticercose no município de Salinas, Minas Gerais.

\section{MATERIAL E MÉTODOS}

Para o estudo da prevalência da cisticercose bovina, teníase humana e avaliação dos fatores de risco associados à manutenção do complexo teníase-cisticercose bovina, foi selecionado o município de Salinas, localizado no norte do estado, na região fisiográfica do chapadão do Itacambira, Vale do Jequitinhonha, a uma altitude máxima de $1.030 \mathrm{~m}$ e a mínima de $432 \mathrm{~m}$, latitude $16^{\circ} 10^{\prime} 19^{\prime \prime}$ sul e longitude $42^{\circ} 17^{\prime} 33^{\prime \prime}$ oeste. 0 município destaca-se como um dos principais polos do desenvolvimento econômico da região, com população estimada de 34.561 habitantes, e destes, 20.692 correspondem à população urbana e 13.869 , à população rural. No município de Salinas, predomina o clima semiárido, com período de seca marcante, temperatura média de $33^{\circ} \mathrm{C}$ no verão e de $18^{\circ} \mathrm{C}$ no inverno, umidade relativa do ar média de 63\% e índice pluviométrico com média anual de 855,0mm (IBGE 2010).

Foi realizado um estudo epidemiológico transversal, no período de janeiro a dezembro de 2010 , que envolveu dezoito propriedades localizadas na área rural do município de Salinas, abrangendo oito comunidades (Quadro 1). Para a seleção das propriedades, optou-se pela amostragem por conglomerado não probabilística. O cálculo amostral foi realizado considerando o número total de bovinos na região, uma prevalência estimada de $2 \%$ de propriedades positivas para a cisticercose, uma precisão de 1,5\% e um nível de significância de 5\%(WHO 2008). Com base nesses critérios, foi obtida uma amostragem mínima de 322 animais, que resultou na seleção de 355 bovinos para coleta de sangue. 0 número de animais selecionados em cada fazenda foi determinado pelo programa Herdacc (Jordan 1995), de acordo com a sensibilidade 
Quadro 1. Relação do número de bovinos por comunidades e propriedades rurais do município de Salinas/MG

\begin{tabular}{lccc}
\hline $\begin{array}{c}\text { Comunidades rurais } \\
\text { do município de } \\
\text { Salinas amostradas }\end{array}$ & $\begin{array}{c}\text { № propriedades } \\
\text { rurais, amostradas } \\
\text { por comunidade }\end{array}$ & $\begin{array}{c}\text { № de bovinos } \\
\text { por comuni- } \\
\text { dade }\end{array}$ & $\begin{array}{c}\text { № de bovinos } \\
\text { amostrados por } \\
\text { comunidade }\end{array}$ \\
\hline 1. Matrona & 1 & 38 & 34 \\
2. Canadá-Matrona & 1 & 39 & 20 \\
3. Jucurutu & 1 & 35 & 30 \\
4. Boqueirão & 1 & 47 & 20 \\
5. Vereda & 1 & 170 & 20 \\
6. Salinas & 1 & 42 & 20 \\
7. Curralinho & 1 & 423 & 60 \\
8. Água Boa & 1 & 192 & 52 \\
9. Novo Horizonte & 1 & 65 & 37 \\
10. Rubelita & 1 & 610 & 62 \\
Total & 18 & 1661 & 355
\end{tabular}

Quadro 2. Número e seleção de propriedades de acordo com o número de bovinos, distribuído em quatro classes funcionais

\begin{tabular}{lccc}
\hline Classes & $\begin{array}{c}\text { Total de } \\
\text { propriedades }\end{array}$ & $\%$ & $\begin{array}{c}\text { № de propriedades } \\
\text { selecionadas }\end{array}$ \\
\hline 250-500 bovinos & 35 & 3,8 & $1(0,68)$ \\
50-250 bovinos & 182 & 19,5 & $4(3,51)$ \\
$20-50$ bovinos & 385 & 41,2 & $7(7,42)$ \\
$<20$ bovinos & 332 & 35,5 & $6(6,39)$ \\
Total & 934 & 100 & 18
\end{tabular}

e especificidade dos testes utilizados, resultando em um número de 20 animais por fazenda, ou a sua totalidade, nos casos de população inferior a 20 animais (Quadro 2).

Em cada propriedade, foi aplicado um questionário semi- estruturado para a obtenção de dados sobre possíveis fatores de risco para a teníase humana e a cisticercose bovina, relacionados ao sistema de criação animal, às condições sanitárias das propriedades e aos hábitos alimentares das famílias (Anexo 1). Foram abordadas questões relativas à fonte de água fornecida às pessoas e animais; sobre o destino do esgoto sanitário; sobre o tratamento térmico e a origem da carne bovina consumida pelas famílias. Foi realizada também a coleta de amostras de fezes dos habitantes das propriedades selecionadas para realização do exame parasitológico para pesquisa de ovos de enteroparasitas por sedimentação espontânea, pelo método de Hoffman, Pons e Janer - HPJ (Hoffman et al. 1934). Além disso, foram analisados dados da prevalência de teníase em Salinas nos anos de 2007, 2008, 2009 e 2010, cedidos pela Secretaria Municipal de Saúde, obtidos a partir de análises realizadas pelo método HPJ.

Para o diagnóstico sorológico da cisticercose bovina, as amostras de sangue dos animais, coletadas por punção da veia jugular, foram mantidas em temperatura ambiente para a separação do soro, e em seguida, armazenadas a $-20^{\circ} \mathrm{C}$. 0 método ELISA indireto foi utilizado como teste de triagem e as amostras positivas foram submetidas ao Immunoblot para a confirmação, de acordo com as metodologias descritas por Pinto et al. (2000) e Pinto et al. (2001), respectivamente. Para as análises pela técnica do Immunoblot, foi realizada a separação dos peptídeos do antígeno vesicular de $T$. crassiceps por eletroforese em gel de gradiente (4$12,5 \%$ ) de poliacrilamida (SDS-PAGE) em sistema descontínuo, segundo Laemmli (1970).

Os resultados das análises laboratoriais e os dados extraídos dos questionários foram armazenados em bancos de dados do Programa Epi info, versão 3.5.1 (WHO 2008). Foram calculadas as médias das variáveis quantitativas, as frequências das variáveis independentes, e a prevalência aparente (PA) e Prevalência real (PR), sensibilidade em série (sensibilidade do teste $1 \times$ sensibili- dade do teste 2 ), a especificidade em série dos testes empregados [1 ( 1 = especificidade do teste 1$) \times 2$ ( 2 = especificidade do teste 2)], de acordo com Martin et al. (1992). Considerou-se o ponto de corte igual a um, significando que o achado de apenas um animal positivo no rebanho já classificava como rebanho positivo. Para verificação de possíveis associações entre as prevalências e os fatores de risco foi calculado o Odds Ratio com intervalo de confiança de 95\% e aplicados os testes Qui-Quadrado ou teste exato de Fisher quando apropriado, em nível de significância de 5\%.

Os objetivos do estudo foram apresentados a todos os participantes e os Termos de Consentimento Livre e Esclarecido (TCLE) individualmente assinados. Este estudo foi aprovado pelo Comitê de Ética em Pesquisa com Animais (Processo 047-2007) e pelo Comitê de Ética em Pesquisa com Seres Humanos (Processo 0752007), vinculados à Universidade Federal de Viçosa.

\section{RESULTADOS E DISCUSSÃO}

Os resultados dos testes de triagem, realizados pelo método ELISA indireto, demonstraram que o número de amostras de soro bovino consideradas suspeitas foi de 52 $(14,68 \%)$, e destas, 16 foram confirmadas como positivas pelo Immunoblot, indicando uma prevalência aparente de $4,51 \%\left(\mathrm{IC}_{95 \%}=2,35\right.$ a 6,66). A prevalência real de cisticercose bovina na zona rural do município de Salinas foi de $4,70 \%\left(\mathrm{IC}_{050}=2,50\right.$ a 6,90). A Organização Pan-Americana de Saúde (OPAS) considera uma região endêmica quando apresenta uma prevalência superior a $5 \%$ de cisticercose bovina (OPAS 1994). Santos et al. (2013), ao realizarem um levantamento soro-epidemiológico da cisticercose bovina no município de Viçosa/MG, utilizando os mesmos testes diagnósticos, encontraram menor prevalência de cisticercose bovina $(0,42 \%)$, quando comparado aos resultados do presente trabalho.

Dados do levantamento epidemiológico de teníase, realizado pela Secretaria Municipal de Saúde de Salinas, revelaram uma prevalência de teníase de $0,29 \%$ no ano de 2007, de $0,36 \%$ em 2008, 0,24\% em 2009 e $0,24 \%$ em 2010. No inquérito coproparasitológico, das 42 amostras de fezes coletadas dos moradores das propriedades rurais avaliadas, nenhuma foi positiva para teníase. No entanto, foram encontradas 37 amostras positivas para outras enteroparasitores, indicando uma prevalência de $88,09 \%$, sendo que $4,76 \%$ das amostras foram positivas para Ascaris lumbricoides, 30,95\% positivas para Entamoeba coli, 33,33\% para Entamoeba histolytica e 23,81\% apresentaram poliparasitismo por Entamoeba coli/Entamoeba histolytica. Segundo Villa (1995), a prevalência de Taenia spp. no Brasil varia de 0,21 a 2,83\%. A menor prevalência de teníase quando comparada a outras parasitoses intestinais também foi observada por outros autores e pode ser explicada pela baixa sensibilidade do método HPJ (Garro et al. 2015). Esta técnica é empregada rotineiramente nos laboratórios de análises clínicas devido à facilidade de execução e baixo custo, porém, devido à grande quantidade de detritos fecais no sedimento, o diagnóstico pode se tornar difícil, principalmente em amostras com baixa carga parasitária (Cantos et al. 2011).

Das propriedades positivas, com relação ao destino dos animais, apenas em $27,7 \%$ das propriedades, os animais eram destinados ao abate em matadouros-frigoríficos, com inspeção sanitária estadual. Torna-se importante ressaltar 
que $22,3 \%$ das propriedades realizavam abate clandestino e que cerca de $1 / 3(33,30 \%)$ das propriedades, embora destinassem os animais para o abate inspecionado, também utilizavam a produção pecuária para consumo próprio, o que aumenta o risco da população em adquirir doenças, como a teníase, ao consumir carne mal-passada e sem inspeção sanitária. Rezende et al. (2006) avaliaram a ocorrência de cisticercose em bovinos abatidos clandestinamente em Silva Jardim/RJ e observaram que, apesar de $21,7 \%$ das carcaças serem positivas, elas eram destinadas ao consumo humano sem nenhum tipo de restrição.

A análise dos dados obtidos pela aplicação dos questionários para caracterização do perfil sanitário e socioeconômico da região demonstrou uma variação na ocorrência dos indicadores pesquisados, que poderiam influenciar no desenvolvimento do complexo teníase-cisticercose. Aproximadamente $66,7 \%$ das famílias possuíam renda mensal de um salário-mínimo (valor de referência: R \$ 510,00 ou US\$ $306,95)$. Ao analisar os fatores que poderiam influenciar no desenvolvimento do complexo teníase-cisticercose em 84 famílias em assentamentos rurais no Pontal do Paranapanema/SP, Prestes-Carneiro et al. (2006) relataram a associação da renda familiar (aproximadamente US\$100,00) à alta prevalência de cisticercose humana $(3,6 \%)$, diagnosticada por meio do teste Immunoblot.

Em relação ao sistema de criação dos bovinos, em todas as propriedades os animais eram criados em sistema extensivo, com adoção de pouca tecnologia. Todas as propriedades positivas para cisticercose bovina forneciam água aos animais sem nenhum tipo de tratamento. Dessas, em $80 \%$ das propriedades a água era proveniente de represa ou lagoa e em $20 \%$ delas, a água fornecida era originada de poço artesiano. Quanto aos fatores de risco envolvidos na transmissão da cisticercose bovina, e citados por alguns autores, destacam-se: o fornecimento de alimentos volumosos ou água contaminados, o consumo de carne crua, a utilização de terras agrícolas para atividades de lazer ou turismo (Abunna et al. 2008). Boone et al. (2007) observaram que o alagamento de pastagens e o livre acesso do gado a águas superficiais (rios, lagos e canais), além da proximidade de uma fonte de efluentes ou águas residuais, são fatores de risco para a ocorrência da cisticercose bovina, reforçando a idéia de que a água desempenha papel importante no transporte de ovos de Taenia saginata.

Quanto ao destino do esgoto sanitário, em 33,33\% das propriedades que apresentaram animais suspeitos no ELISA, o esgoto era lançado a céu aberto e, em $20 \%$, em fossa séptica. Em todas as propriedades, as fossas localizavam-se perto da residência (menos de 10 metros) e de cultura de horta. Ao contrário do que foi observado no presente estudo, o esgoto lançado a céu aberto tem sido considerado um importante fator na disseminação deste complexo por permitir o contato dos animais com excretas humanos inadequadamente tratados (Rezende et al. 2006, Falavigna-Guilherme et al. 2007).

Após uma simulação da Odds Ratio dos fatores de risco para a transmissão do complexo teníase-cisticercose, considerando a prevalência encontrada de cisticercose bovina (Quadro 3), verificou-se que apenas a variável tratamento
Quadro 3. Análise de associação entre os possíveis fatores de risco para a transmissão do Complexo Teníase-Cisticercose, no município de Salinas. MG

\begin{tabular}{lr}
\hline $\begin{array}{l}\text { Possíveis fatores de risco para transmissão } \\
\text { complexo teníase-cisticercose }\end{array}$ & Odds Ratio \\
\hline Fonte de água fornecida aos animais: & \\
$\quad$ Poço Artesiano & $1,00^{\mathrm{a}}$ \\
$\quad$ Rio/Ribeirão & 0,48 \\
$\quad$ Represa & 0,81 \\
Fonte de água consumo humano: & $1,00^{\mathrm{a}}$ \\
$\quad$ COPASA & 0,59 \\
Poço Artesiano & 3,14 \\
$\quad$ Rio/Ribeirão & 0,00 \\
$\quad$ Represa & \\
Destino Esgoto: & $1,00^{\mathrm{a}}$ \\
$\quad$ Fossa Séptica & 3,83 \\
$\quad$ Céu Aberto & \\
Tratamento térmico da carne: & $1,00^{\mathrm{a}}$ \\
$\quad$ Bem Passada & $3,58^{*}$ \\
$\quad$ Malpassada & \\
Origem da carne bovina consumida pelas famílias: & $1,00^{\mathrm{a}}$ \\
$\quad$ Comércio & 2,51
\end{tabular}

${ }^{a}$ Valor de referência para cálculo de OddsRatio. Para calculo do OR foi fixado o fator de menor risco, atribuindo para tal risco 1,00 e posteriormente foi calculado o OR das outras variáveis tomando como referência a variável de menor risco; b COPASA = Companhia de Saneamento de Minas Gerais. * Diferença estatística em relação aos demais valores.

térmico da carne bovina indicou uma diferença significativa, demonstrando que os indivíduos expostos ao consumo de carne bovina mal-passada têm 3,58 mais chances de adoecer que os indivíduos pertencentes ao grupo não exposto a este fator.

A ingestão de carne crua ou malcozida contendo cisticercos viáveis é a principal fonte de transmissão da teníase ao hospedeiro definitivo, o homem, mantendo o ciclo biológico da doença no ambiente (Silva \& Silva 2007, Ungar et al. 2008). Observou-se ainda a relevância do fato de que os entrevistados assumiram consumir carne mal-passada em $40 \%$ das propriedades positivas. Este fato foi descrito por Wandra et al. (2006), ao estudarem a prevalência de $T$. saginata em Bali, na Indonésia, onde todos os indivíduos positivos para teníase revelaram consumir carne bovina crua. Todos os entrevistados das propriedades positivas para cisticercose bovina alegaram saber "o que é a doença popularmente conhecida como canjiquinha ou pipoca" e já terem visto material contaminado. Dos que já a tinham visto, $20 \%$ relataram tê-la visto em sua propriedade e $80 \%$, em outras propriedades e no comércio regional, principalmente no Mercado Municipal de Salinas.

Os resultados encontrados no presente estudo reforçaram a necessidade de implantação do controle higiênico-sanitário e tecnológico (HST) na obtenção da carne bovina no município de Salinas/MG. Além dessa medida, é necessária eliminação do abate não inspecionado, pois o consumo de carne malpassada e não inspecionada foi considerado o principal fator de risco para a ocorrência e a manutenção do complexo teníase-cisticercose bovina. 0 controle do comércio varejista de carnes e derivados pela vigilância sanitária municipal também é importante para 
impedir a comercialização de carnes não inspecionadas, infectadas com Cysticercus bovis, que representam um risco para a saúde do consumidor.

\section{CONCLUSÃO}

Conclui-se que a cisticercose bovina está presente no município de Salinas, Minas Gerais, sendo o tratamento térmico ineficiente da carne bovina o principal fator de risco para a manutenção do complexo teníase-cisticercose, o que reforça a necessidade da adoção de medidas de controle com contínua vigilância epidemiológica e sanitária.

Agradecimentos.- Os autores agradecem à Fundação de Apoio à Pesquisa do Estado de Minas Gerais (FAPEMIG), ao Conselho Nacional de Desenvolvimento Científico e Tecnológico (CNPq), ao Ministério da Agricultura, Pecuária e Abastecimento (MAPA) por fornecerem fundos para realização desta pesquisa, ao Programa de Pós-Graduação da Escola de Veterinária da UFMG, ao IFNMG, Câmpus Salinas, à Prefeitura Municipal de Salinas e ao Instituto Mineiro de Agropecuária (IMA), escritório de Salinas.

\section{REFERÊNCIAS}

Abunna F., Tilahun G. \& Bersissa K.A. 2008. Bovine cysticercosis in Awassa, southern Ethiopia: prevalence, risk factors and cyst viability. Zoonoses and Public Health 55:82-88.

Almeida L.P., Moreira M.D., Reis D.O. \& Santos W.L.M. 2002. Cisticercose bovina: um estudo comparativo entre animais abatidos em frigoríficos com serviço de Inspeção Federal e com Inspeção Municipal. Hig. Aliment. 16:51-55.

Boone I., Thys E., Marcotty T., Borchgrave J., Ducheyne E. \& Dorny P. 2007. Distribution and risk factors of bovine cysticercosis in Belgian dairy and mixed herds. Prev. Vet. Med. 82:1-11.

Cantos G.A., Galvão M. \& Linécio J. 2011. Comparação de métodos parasitológicos tendo como referencial o método de Faust para a pesquisa de cistos de protozoário. NewsLab 104:160-165.

IBGE 2010. Censo Demográfico. Instituto Brasileiro de Geografia e Estatística. Disponível em <http://censo2010.ibge.gov.br> Acesso em 1 set. 2015.

Dorny P., Brandt J., Zoli A. \& Geerts S. 2003. Immunodiagnostic tools for human and porcine cysticercosis. Acta Trop. 87(1):79-86.

Esteves F.M., Silva-Vergara M.L. \& Carvalho A.B. 2005. Inquérito epidemiológico sobre teníase em população do Programa Saúde da Família no Município de Uberaba, MG. Revta Soc. Bras. Med. Trop. 38(6):530-531.

Falavigna-Guilherme A.L., Silva K., Araujo S.M., Tobias M.L. \& Falavigna D.L.M. 2007. Cisticercose em animais abatidos em Sabáudia, Estado do Paraná. Arq. Bras. Med. Vet. Zootec. 58(5):950-951.

Flisser A., Sarti E., Lighowlers M. \& Schantz P. 2003. Neurocysticercosis: regional status, epidemiology, impact and control measures in the Americas. Acta Trop. 87(1):43-51.

Garro F.L., Santos T.M., Assis D.C.S., Heneine L.G.D., Ornellas C.B.D., Pinto P.S.A. \& Santos W.L.M. 2015. Diagnóstico do complexo teníase-cisticercose bovina em São João Evangelista, Minas Gerais, Brasil. Arq. Bras. Med. Vet. Zootec. 67(4):1063-1069.

Girotto A., Pinto P.S.A., Dias J.C.O., Chaves L.S. \& Ferreira H.C.C. 2009. Detecção de peptídeos importantes para o diagnóstico da cisticercose bovina no Imunoblot. Ciência Rural 39(4):1136-1140.

Hoffman W.A., Pons J.A. \& Janer S.L. 1934. The sedimentation concentration method in Schistosomiasis mansoni. Puerto Rico J. Publ. Health 9:283-291.

Jordan D. 1995. Herdacc: A Programme for Calculating Herd Level (aggregate) Sensitivity and Specificity. Department of Population Medicine, University of Guelph, Guelph, Canada.

Khaniki G.R., Raei M., Kia E.B., Haghi A.M. \& Selseleh M. 2010. Prevalence of bovine cysticercosis in slaughtered cattle in Iran. Trop. Anim. Health Prod. 42(2):141-143.
Laemmli U.K. 1970. Cleavage of structural proteins during the assembly of the head of bacteriophage T4. Nature 227(5259):680-685.

Marques G.M., Buzi K.A., Galindo L.A., Baldini E.D. \& Biondi G.F. 2008. Avaliação dos registros de condenação por cisticercose em bovinos abatidos em frigoríficos da região centro oeste do estado de São Paulo (19962000). Vet. Zootec. 15(1):114-120.

Martin S.W., Shoukri M. \& Thorburn M.A. 1992. Evaluating the health status of herds based on tests applied to individuals. Prev. Vet. Med. 14(1):33-43.

Mendes E.C., Silva S.S., Fonseca E.A.L.T., Souza H.R.R. \& Carvalho R.W. 2005. A neurocisticercose humana na baixada fluminense, estado do Rio de Janeiro, Brasil. Arqs Neuro-Psiquiatria 63(4):1058-1062.

Monteiro L.L., Pinto P.S.A., Salcedo J.H.P., Araújo J.V., Santos W.L.M. \& Cecon P.R. 2007. Taenia solium metacestode antigens in ELISA for the diagnosis of bovine cysticercosis. Arq. Bras. Med. Vet. Zootec. 59(1):21-25.

Moreira M.D., Almeida L.A., Reis D.O. \& Santos W.L.M. 2002. Cisticercose bovina: um estudo com bovinos abatidos em Matadouro Municipal de Uberlândia, MG. Hig. Aliment. 16(100):37-42.

OPAS 1994. Epidemiologia y Control de la Teniasis/Cisticercosis en America Latina. 3rd ed. Organizacion Pan-Americana de la Salud - OPAS/ OMS, Washington, DC. 297p.

Pinto P.S.A., Vaz A.J., Germano P.M.L. \& Nakamura P.M. 2000. Elisa test for the diagnosis of cysticercosis in pigs using antigens of Taenia solium and Taenia crassiceps cysticerci. Revta Inst. Med. Trop. São Paulo 42(2):7179.

Pinto P.S.A., Vaz A.J., Nakamura P.M. \& Germano P.M.L. 2001. Immunoblot analisys using antigens from Taenia crassiceps cysticerci in the diagnosis of swine cysticercosis. Boln Chil. Parasitol. 56(1/2):36-42.

Prestes-Carneiro L.E., Freitas S.B.Z., Zago S.C.S., Miguel N.A., Primo O.B., Iha A.H., Espíndola N.M. \& Vaz A.J. 2006. Taeniosis-cysticercosis complex in individuals of a peasants' settlement (Teodoro Sampaio, Pontal of Paranapanema, SP, Brazil). Mem. Inst. Oswaldo Cruz 101(1):15-20.

Reis D.O., Mundim M.J.S., Cabral O.D. \& Costa-Cruz J.M. 1996. Cisticercose bovina: 15 anos de ocorrência em animais abatidos em Uberlândia, Minas Gerais, Brasil (1979-1993). Hig. Aliment. 10(43):33-35.

Rezende R.B.C., Fernandez A.T., Costa F. \& Silva T.J.P. 2006. Ocorrência de cisticercose em bovinos abatidos clandestinamente no município de Silva Jardim, RJ. Hig. Aliment. 21(140):103-109.

Santos T.O., Pinto P.S.A., Iasbik A.F., Silva L.F., Acevedo Nieto E.C. \& Guimarães-Peixoto R.P.M. 2013. Epidemiological survey of the taeniasis/cysticercosis complex in cattle farms in Viçosa County, Minas Gerais, Brazil. Pesq. Vet. Bras. 33(4):449-452.

Sato M.O., Yamasaki H., Sako Y., Nakao M., Nakaya K., Plancarte A., Kassuku A.A., Dorny P., Geerts S., Benitez-Ortiz W., Hashiguchi Y. \& Ito A. 2003. Evaluation of tongue inspection and sorology for diagnosis of Taenia solium cysticercosis in swine: usefulness of ELISA using purified glycoproteins and recombinant antigen. Vet. Parasitol. 111(4):309-322.

Silva A.A.P. \& Silva M.V. 2007. Teníase na população do bairro Nossa Senhora Aparecida município de Correia Pinto-SC, em 2003 e 2004. Revta Bras. Anal. Clín. 39:143-145.

Ungar M.L., Germano P.M.L. \& Germano M.I.S. 2008. Cisticercose Bovina, p.449-461. In: Germano P.M.L. \& Germano M.I.S. (Eds), Higiene e Vigilância Sanitária de Alimentos. 3aㅡ ed. Manole, São Paulo.

Vieira N.P., Faria P.B., Matos M.R. \& Pereira A.A. 2011. Condenação de fígados bovinos na região sul do estado do Espírito Santo. Arq. Bras. Med. Vet. Zootec. 63(6):1605-1608.

Villa M.F.G. 1995. Situação epidemiológica do complexo teníase-cisticercose como problema de saúde pública no Brasil. Hig. Aliment. 9(36):8-11.

Wandra T., Depary A.A., Sutisna P., Margono S.S., Suroso T., Okamoto M., Craig P.S. \& Ito A. 2006. Taeniasis and cysticercosis in Bali and North Sumatra, Indonesia. Parasitol. Int. 55(1):155-160.

WHO 2008. World Health Association EPI INFO' ${ }^{\text {TM }}$. Current/Version: Version 3.5.1 Centers for Disease Control and Prevention (CDC). Disponível em < http://www.cdc.gov/epiinfo/epiinfo.htm> Acesso em 23 abr. 2014. 